\title{
Evaluation of sperm chromatin structure and DNA strand breaks is an important part of clinical male fertility assessment
}

\author{
Donald P. Evenson ${ }^{1,2,3}$ \\ ${ }^{1}$ South Dakota State University, Brookings, SD, USA; ${ }^{2}$ Department of OB/GY, Sanford Medical School, University of South Dakota, Sioux Falls, \\ SD, USA; ${ }^{3}$ SCSA Diagnostics Inc., Brookings, SD, USA \\ Correspondence to: Donald P. Evenson. President and Director, SCSA Diagnostics Inc., $3026^{\text {th }}$ St. W., Brookings, SD 57006, USA. Email: don@scsatest.com. \\ Comment on: Agarwal A, Majzoub A, Esteves SC, et al. Clinical utility of sperm DNA fragmentation testing: practice recommendations based on \\ clinical scenarios. Transl Androl Urol 2016;5:935-50.
}

Submitted May 21, 2017. Accepted for publication Jul 12, 2017.

doi: $10.21037 /$ tau.2017.07.20

View this article at: http://dx.doi.org/10.21037/tau.2017.07.20

\section{Introduction}

As reviewed by Agarwal et al. (1) and recently stated by $\mathrm{Vu}$ Bach and Schlegel (2), "numerous studies have attempted to assess the association between elevated sperm DNA fragmentation (SDF) and ART outcomes. Unfortunately, variations between SDF assays, protocols, and thresholds and differences in study populations have resulted in systematic reviews and meta-analyses fraught with heterogeneity and unable to come to robust conclusions". However, these early meta-analyses were considered a near impossible task leading to vague and questionable conclusions. Recent studies have refined protocols for the Sperm Chromatin Structure Assay (SCSA) $(3,4)$ TUNEL (5), SCD (6) and COMET (7) tests. A major consideration for standardizing a protocol is to identify the SDF thresholds. While this has often been described as a single\% SDF, e.g., 30\% listed as the SCSA threshold in Agarwal et al. (1), more recent SCSA studies have listed two or three thresholds depending on the method of fertilization (see below).

\section{SDF tests}

The authors (1) provided eight protocols to measure sperm DNA/chromatin integrity. The first shown was the light microscope "AO test" that uses acridine orange (AO) to determine the percent (\%) of sperm in a semen sample that fluoresce red (broken DNA) or green (intact DNA) (8). This test is considered unreliable for the sensitive human clinic (9).

In 1980, the first SDF test, the SCSA ${ }^{\circledR}$ was introduced (10).
A very significant advantage of the SCSA test is that the marker for DNA strand breaks is the very small, flat planer AO molecule (MW 265). Thus, AO likely penetrates the entire highly compact nuclear chromatin structure $(9,11)$. In contrast, the TUNEL assay requires the large terminal deoxynucleotidyl transferase (TdTA) enzyme to label at sites of DNA strand breaks, except those breaks without a 3' $\mathrm{OH}$ end (12). It is likely the protamine toroid is not penetrable by this enzyme, thus reducing the efficiency of flow cytometric TUNEL testing by $1 / 3(9,11)$.

\section{What does the SCSA test measure?}

Agarwal et al. (1) have stated that the SCSA test "measures the susceptibility of DNA to denaturation, which occurs more commonly in fragmented DNA". Also, a recent review (2) stated that the "SCSA starts with an acid denaturation step and depends on the principle that abnormal DNA is more prone to further fragmentation by acid denaturation than intact DNA". Does that imply that the acid causes fragmentation leading to DNA strand breaks? All data to date strongly suggest that the function of the heat or $\mathrm{pH}$ 1.20 treatment for 30 secs is to denature (open) the two DNA strands at the sites of existing single or double DNA strand breaks, i.e., "normal DNA" with single or double strand breaks in the phosphodiester backbone of DNA (13). Since neither heat $\left(100{ }^{\circ} \mathrm{C}, 5 \mathrm{~min}\right)$ nor acid $(\mathrm{pH} 1.20 / 30$ sec) (13) break the DNA phosphodiester backbone, both the TUNEL and the SCSA tests are likely measuring existing DNA breaks available to each specific molecular probe (9). 

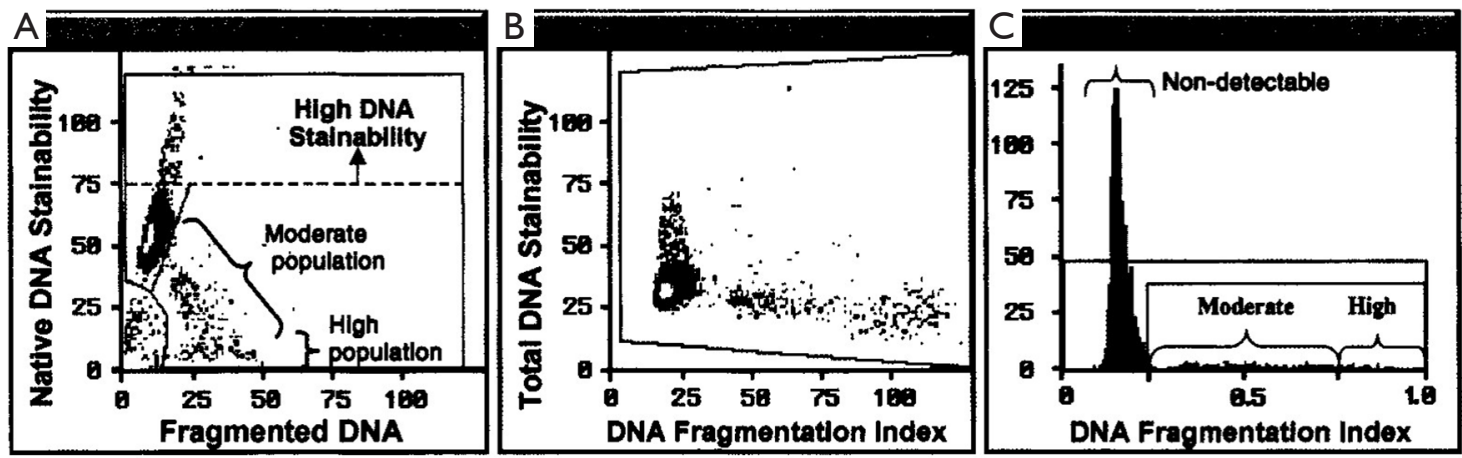

Figure 1 SCSA data for clinical report. (A) Raw data from a flow cytometer showing each of 5,000 sperm as a single dot on a scattergram. $\mathrm{Y}=$ native DNA stainability, $\mathrm{X}=$ fragmented DNA; high DNA stainability (HDS) sperm with uncondensed chromatin; (B) $\mathrm{SCSA}_{\text {soft }}{ }^{\circledR}$ software conversion to: $\mathrm{Y}=$ total DNA stainability, $\mathrm{X}=\mathrm{DNA}$ fragmentation index; (C) frequency histogram of data in middle panel. Three levels of DNA fragmentation: non-detectable, moderate level and high level. SCSA, Sperm Chromatin Structure Assay.

\section{Flow cytometry (FCM) SDF tests: SCSA and TUNEL}

Of critical importance for validation of any test is its precision and accuracy. Without a doubt, FCM is invaluable to achieve this requirement for the SCSA and TUNEL tests. Measurement by using FCM is highly rapid with exceptional mechanical precision that avoids human eye biases. Both the SCSA and the TUNEL test measure five to ten thousand single cells, one at a time, at rates of about $250 \mathrm{cells} / \mathrm{sec}(14)$. SCSA has a significant advantage of being a dual parameter measurement. Thus, each spermatozoon is characterized by $1,024 \times 1,024$ units (channels) of green $v s$. red fluorescence. Data are presented as a dot plot (one dot for each of 5,000 sperm) with both $\mathrm{X}$ and $\mathrm{Y}$ axes of 1,024 channels or 1,024 degrees of DNA damage (14) as seen in Figure 1.

The SCSA test measures two sperm nuclear parameters simultaneously: (I) sperm DNA strand breaks (\%DFI); and (II) uncondensed chromatin [\% high DNA stainability (HDS)] (15). AO stains histone complexed DNA 2.3× more than protamine complexed DNA (16) and is clearly resolved as seen in Figure 1.

From SCSA scatterplots, in live time, populations of normal, increased red fluorescence and increased green fluorescence can be FCM-sorted out into test tubes for further morphological or biochemical analyses (17). Normal and moderate level DFI populations have normal morphology while the high DFI populations have abnormal morphology (17) consistent with apoptotic sperm.

Both DFI populations have $\mathrm{pH} 10$ Comet positivity (double strand breaks) confirming that SCSA DFI populations have Comet-confirmed sperm DNA strand breaks (17). The sperm nuclei of the HDS population are more rounded consistent with morphological immature sperm and no Comets $(17,18)$. For the SCSA test, raw or extended aliquots of semen (fresh or frozen) are sent to a diagnostic center on dry ice or in LN2 dry shippers. Immediately after thawing, the sample is treated with a low $\mathrm{pH}$ buffer ( $\mathrm{pH} 1.20,30 \mathrm{sec})$, stained with $\mathrm{AO}$ and immediately measured (14). SCSA data clearly show that these frozen and rapidly thawed samples have the exact same SCSA values as fresh samples $(19,20)$.

\section{Measures of uncondensed chromatin (\%HDS)}

HDS sperm have uncondensed chromatin easily detectable by SCSA since more ds DNA is exposed to AO staining. The final structure of sperm chromatin is dependent upon post-translational methylation and acetylation that affects chromatin stability and the acquisition of epigenetic/imprinting marks impacting on embryonic development. This faulty compaction makes an abnormal tertiary chromatin structure that is crucial for correct timing during the process of fertilization and early preimplantation development $(21,22)$. The threshold for HDS related embryo failure is $20 \%$ to $>25 \%$ HDS; $22 \%$ of 1,417 infertility patients were at these levels (23).

\section{Repeatability of multi-lab flow cytometric measures of human clinical samples}

Now that flow cytometers are available in numerous laboratories and medical institutions around the world, it 


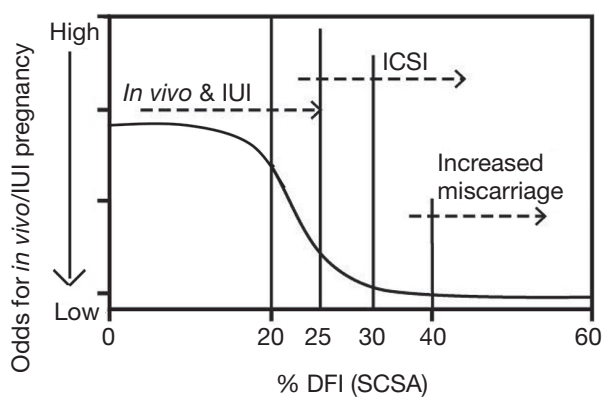

Figure 2 Odds of in vivo/IUI pregnancy $v s$. \%DFI. The curve of decreasing odds with increasing \% DFI is drawn from data on semen samples used for in vivo $(\mathrm{n}=732)(10,27,28)$ and IUI $(\mathrm{n}=387)(29)$ clinical studies. Vertical lines between 20-40 \% DFI indicate suggested clinical intervention thresholds from data $(n \geq 1,500$ semen samples) in ART clinical studies [e.g. $(23,29,30)]$.

is very important to know whether multiple types of flow cytometers are applicable to measure SDF in the SCSA and TUNEL tests. In 1995, Evenson and ten collaborators in seven centers on two continents made comparative SCSA \%DFI measurements of aliquots of the same frozen samples from human, mouse, rat, turkey, bull, ram, boar and stallion (24). Both epiillumination and orthogonal optic flow cytometers were compared. Of great importance, the overall \%DFI values for the total 132 samples had correlations of $0.9886(\mathrm{P}<0.001)$. This number solidly demonstrates that the crucial SCSA measurements around the world on very different flow cytometers produced with SCSA software (or equivalent red/red + green fluorescence) produced the near exact results. Similarly, Ribeiro et al. (25) have recently shown that TUNEL measurements on two continents gave the same data.

Comparative measurements of aliquots of human patient semen samples by SCSA Diagnostics, Inc. (SDI) personnel and SDI trained FCM operators at the University of Copenhagen (10 samples; $\left.\mathrm{R}^{2}=0.9812\right)$ and the Andrology Lab, Coimbatore, India $(57$ samples; $\mathrm{R}^{2}=0.962$ ) (9) showed that the near exact SCSA data can be obtained between labs.

\section{SCSA data and pregnancy outcomes are predictive of male infertility via intercourse, IUI, IVF and ICSI}

The major use of many thousands of SCSA tests has been to determine the \%DFI, i.e., the percent of sperm in a population that has measurable single or double strand
DNA breaks of the phosphodiester DNA backbone. This has also been called "sperm with fragmented DNA (Latin fragmentum: a broken piece), thus, DNA with pieces of broken single or double DNA strands.

\section{Pioneer in vivo male factor studies}

The first well executed clinical in vivo study correlating sperm DNA integrity with pregnancy outcome was done in collaboration with Mike Zinaman at Georgetown University (26). The SCSA test was used to measure human semen samples from 165 presumably fertile couples wishing to achieve pregnancy over twelve menstrual cycles. Any woman with female infertility factors was excluded. SCSA data from the male partners of 73 couples (group 1) achieving pregnancy during months 1-3 were compatible with "high fertility". These SCSA values were significantly different from those of 40 couples (group 3) achieving pregnancy in months $4-12(\mathrm{P}<0.01)$ and of those male partners of 31 couples (group 4 ) not achieving pregnancy $(\mathrm{P}<0.001)$. Group 2 included couples who had a miscarriage. "Based on logistic regression, the level of \%DFI was the best predictor for whether a couple would not achieve pregnancy". Some $84 \%$ of males in group 1 had $<15 \%$ DFI; no couples achieved pregnancy in group 1 with $>30 \%$ DFI.

Shortly after the above publication, Spanò et al. (27) published a "time to natural pregnancy" on 215 "Danish first pregnancy planners" with no previous knowledge of their fertility status. SCSA data was obtained on 1,301 cycles (838 cycles, months 1-6; 463 cycles, months 7-24). At $20 \%$ DFI, fecundability started dropping and became very small for values of $30-40 \%$ as seen in Figure 2.

Thus, the probability of fathering a child sharply declined beginning at $20 \%$ DFI and was negligible when this fraction added up to $40 \%$. As stated by the authors, "this level makes this individual a good candidate not to conceive". The results of both above studies are consistent with the finding that sperm chromatin structure is reflective of fertility potential, which significantly deteriorates when $\%$ DFI is $>30 \%$. The Evenson study (26) and the Spano study (27) had ORs of 6-7 (28) for higher probability of pregnancy when DFI $<30 \%$ compared with $\mathrm{DFl}>30 \%$.

\section{SCSA and the ART clinic}

\section{IUI}

Bungum et al. (29) studied a total of 998 cycles (387 
IUI, $388 \mathrm{IVF}$ and $223 \mathrm{ICSI}$ ). Of great interest was the observation that when the SCSA \%DFI value was greater than $30 \%$, the IUI pregnancy rate was a dramatically low $1.5 \%$ in contrast to those with DFI $<30 \%$ who had a successful pregnancy rate of $19.0 \%$ (29). These data strongly suggested that men with a DFI of $>30 \%$ had a very low chance with both natural and IUI conception and should move to ICSI. Considering the above data, the threshold for IUI and natural fertility has been set at $25 \%$ DFI since this level is at the statistical limit for significant loss of rapid and successful pregnancy.

\section{IVF and ICSI}

A recent SCSA study by Oleszczuk et al. (30) included 1,633 IVF or ICSI cycles. DFI values were categorized into four intervals: DFI $\leq 10 \%$ (reference group), $10 \% \leq$ DFI $\leq 20 \%$, $20 \%<$ DFI $\leq 30 \%$, DFI $>30 \%$. For the three latter intervals, the following outcomes of IVF/ICSI procedures were analyzed in relation to the reference group: fertilization, good quality embryo, pregnancy, miscarriage, and live births. In the standard IVF group, a significant negative association between DFI and fertilization rate was found. When calculated per ovum pick up (OPU), odds ratios (ORs) for at least one good quality embryo (GQE) were significantly lower in the standard IVF group if DFI $>20 \%$. OR for live birth calculated per OPU was significantly lower in standard IVF group if DFI >20\% (OR, 0.61; 95\% CI: $0.38-0.97 ; \mathrm{P}=0.04)$. No such associations were seen in the ICSI group. OR for live birth by ICSI compared to IVF were statistically significantly higher for DFI $>20 \%$ (OR, 1.7; 95\% CI: 1.0-2.9; $\mathrm{P}=0.05$ ). OR for miscarriage was significantly increased for DFI $>40 \%$ (OR, 3.8; $95 \%$ CI: $1.2-12 ; \mathrm{P}=0.02)$. The results suggest that ICSI might be a preferred method of in vitro treatment in cases with high DFI. Extensive SCSA data on infertility patients have shown that when a patient has $<20 \%$ DFI, such semen sample with regards to sperm DNA integrity is consistent with normal pregnancy by intercourse or IUI unless other classical semen analysis shows one or two abnormal scores which decrease the odds for pregnancy (29,30). Decreasing odds are present with $>20 \%$ DFI and at $25 \%$ DFI the odds become poor for pregnancy by intercourse or IUI. At $30 \%$ DFI, reasonable success requires ICSI. And at 40\% DFI the odds become very poor for pregnancy and increased odds for miscarriage. Values above $50 \%$ may rarely achieve pregnancy, but the odds are indeed poor (30). Figure 2 graphically summarizes the three thresholds for SCSA: (I)
$<20 \%$; (II) $>25 \%$; (III) $30-40 \%$. The $20-25 \%$ DFI has been considered the "grey zone" by Spano et al. (27), Erenpreiss et al. (31), Oleszczuk et al. (30) and Hamadi et al. (21) and fertility problems may start to occur when SCSA DFI reaches this level. It is noted, however, that our SDI clinical service has seen natural full-term pregnancy with up to $68 \%$ DFI. The observation illustrated that these clinical thresholds are statistical values and not absolute values.

The striking observation in Figure 2 is that as little of $5 \%(20-25 \%)$ DFI units, and certainly $10 \%$ (20-30\%) DFI units, have different odds for success. This demands that SDF tests deliver the highest levels of precision, accuracy, and repeatability.

Despite the greater cost of flow cytometric (SCSA, TUNEL) tests, this high precision is obtained by FCM in contrast to potential human eye error with light microscopy. Each clinic must decide the cost/benefit ratio from selection of SDF tests.

\section{Conclusions}

Agarwal et al. (1) reviewed the evolution of SDF tests from their origin to current utility in the urology and infertility clinics. The recognition of SDF testing as a valuable tool for male fertility evaluation has been acknowledged. For the past decade, the American Society of Reproductive Medicine (ASRM) consensus on SDF has indicated an increased potential for clinical use but note that meta- analyses have been fraught with the complexities of four major tests done with different protocols in multiple labs and different clinical thresholds, thus causing a near impossible consensus on their overall utility. Agarwal et al. (1) have brought an updated and a clearer picture on the utility of SDF tests and noted that the latest American Urological Association (AUA) and the European Association of Urology (EAU) guidelines have acknowledged the importance of DNA fragmentation in sperm as assessment of male infertility. This review (l) provides clinical scenarios where SDF testing is important. The positive utility of SDF testing on clinical varicocele patients was a primary focus on this study. Of greater impact, the current utility of SDF testing in the infertility clinic was highlighted focusing on their role in the ART clinic with specific emphasis on strongly recommending SDF testing in patients with recurrent ART failure.

Agarwal et al. (1) concluded their review with the statement: "SDF testing should be included in the evaluation of male factor fertility along with the standard semen analysis". This concurs with the summary of Simon 
et al. (32) "There is sufficient evidence in the existing literature suggesting that sperm DNA damage has a negative effect on clinical pregnancy following IVF and/ or ICSI treatment". Any couple that fails to obtain a pregnancy within a year would gain a valuable insight into the potential that couple infertility may be due to SDF and, if so, to proceed with recommendations presented here (1) to reduce SDF by lifestyle changes or select an ART procedure in part determined by the results of the SDF test.

\section{Acknowledgements}

None.

\section{Footnote}

Conflicts of Interest: The author has no conflicts of interest to declare.

\section{References}

1. Agarwal A, Majzoub A, Esteves SC, et al. Clinical utility of sperm DNA fragmentation testing: practice recommendations based on clinical scenarios. Transl Androl Urol 2016;5:935-50.

2. Vu Bach P, Schlegel PN. Sperm DNA damage and its role in IVF and ICSI. Basic Clin Androl 2016;26:15.

3. Evenson D. Sperm Chromatin Structure Assay (SCSA®): Detailed Protocol. In: Zini A, Agarwal A editors. Sperm Chromatin: Biological and Clinical Applications in Male Infertility and Assisted Reproduction. New York: Springer Sciences, 2011:487-97.

4. Evenson DP. Sperm Chromatin Structure assay (SCSA®): Evolution from origin to clinical utility. In: A Zini A, Agarwal A. editors. A Clinician's Guide to Sperm DNA and Chromatin Damage. New York: Springer Science, 2017.

5. Sharma R, Ahmad G, Esteves SC, et al. Terminal deoxynucleotidyl transferase dUTP nick end labeling (TUNEL) assay using bench top flow cytometer for evaluation of sperm DNA fragmentation in fertility laboratories: protocol, reference values, and quality control. J Assist Reprod Genet 2016;33:291-300.

6. Gosálvez J, López-Fernández C, Fernández JL. Sperm Chromatin Dispersion Test: Technical Aspects and Clinical Applications. In: Zini A, Agarwal A. editors. Sperm Chromatin. New York: Springer, 2011:151-70.

7. Simon L, Carrell DT. Sperm DNA damage measured by comet assay. Methods Mol Biol 2013;927:137-46.
8. Tejada RI, Mitchell JC, Norman A, et al. A test for the practical evaluation of male fertility by acridine orange (AO) fluorescence. Fertil Steril 1984;42:87-91.

9. Evenson DP. The Sperm Chromatin Structure Assay $\left(\mathrm{SCSA}\left({ }^{\circledR}\right)\right)$ and other sperm DNA fragmentation tests for evaluation of sperm nuclear DNA integrity as related to fertility.Anim Reprod Sci 2016;169:56-75.

10. Evenson DP, Darzynkiewicz Z, Melamed MR. Relation of mammalian sperm chromatin heterogeneity to fertility. Science 1980;210:1131-3.

11. Gawecka JE, Boaz S, Kasperson K, et al. Luminal fluid of epididymis and vas deferens contributes to sperm chromatin fragmentation. Hum Reprod 2015;30:2725-36.

12. Gorczyca W, Traganos F, Jesionowska H, et al. Presence of DNA strand breaks and increased sensitivity of DNA in situ to denaturation in abnormal human sperm cells: analogy to apoptosis of somatic cells. Exp Cell Res 1993;207:202-5.

13. Darzynkiewicz Z, Traganos F, Sharpless T, et al. Thermal denaturation of DNA in situ as studied by acridine orange staining and automated cytofluorometry. Exp Cell Res 1975;90:411-28.

14. Evenson DP, Larson KL, Jost LK. Sperm chromatin structure assay: its clinical use for detecting sperm DNA fragmentation in male infertility and comparisons with other techniques. J Androl 2002;23:25-43.

15. Evenson DP, Jost LK, Corzett M, et al. Characteristics of human sperm chromatin structure following an episode of influenza and high fever: a case study. J Androl 2000;21:739-46.

16. Evenson DP, Darzynkiewicz Z, Jost L, et al. Changes in accessibility of DNA to various fluorochromes during spermatogenesis. Cytometry 1986:7:45-53.

17. Evenson DP, Tritle D. Platform Presentation Abstract: "Characterization of SCSA Resolved Sperm Populations by Comet Assay and Image Analysis". IFFS 8th World Congress on Fertility and Sterility. Palais des congres de Montreal, Montreal, Quebec Canada, 2004 May 23/28.

18. Evenson D. Sperm Chromatin Structure Assay (SCSA): 30 years' experience with the SCSA. In: Agarwal A, Zini A. editors. Sperm DNA and Male Infertility and ART, New York, Springer Publishers, 2011:125-49.

19. Evenson DP, Baer RK, Jost LK. Long-term effects of triethylenemelamine exposure on mouse testis cells and sperm chromatin structure assayed by flow cytometry. Environ Mol Mutagen 1989;14:79-89.

20. Evenson DP, Thompson L, Jost L. Flow cytometric evaluation of boar semen by the sperm chromatin 
structure assay as related to cryopreservation and fertility. Theriogenology 1994;41:637-51.

21. Hamidi J, Frainais C, Amar E, et al. A double-blinded comparison of in situ TUNEL and aniline blue versus flow cytometry acridine orange for the determination of in situ TUNEL and aniline blue versus flow cytometry acridine orange for the determination of sperm DNA fragmentation and nucleus decondensation state index. Zygote 2015;23:556-62.

22. Menezo Y. Association between the MTHFR-C677T isoform and structure of sperm DNA. J Assist Reprod Genetics 2017. [Epub ahead of print].

23. Menezo Y, Clement P, Amar E. Evaluation of sperm DNA structure, fragmentation and decondensation: an essential tool in the assessment of male infertility. Transl Androl Urol 2017;6:S553-6.

24. Evenson D, Jost L, Gandour D, et al. Comparative sperm chromatin structure assay measurements on epiillumination and orthogonal axes flow cytometers. Cytometry 1995;19:295-303.

25. Ribeiro S, Sharma R, Gupta S, et al. Inter- and intralaboratory standardization of TUNEL assay for assessment of sperm DNA fragmentation. Andrology 2017;5:477-85.

26. Evenson DP, Jost LK, Marshall D, et al. Utility of the sperm chromatin structure assay as a diagnostic and prognostic tool in the human fertility clinic. Hum Reprod 1999;14:1039-49.

27. Spanò M, Bonde JP, Hjøllund HI, et al. Sperm chromatin damage impairs human fertility. The danish first pregnancy planner study team. Fertil Steril 2000;73:43-50.

28. Evenson DP, Wixon R. Data analysis of two in vivo fertility studies using SCSA derived DNA Fragmentation Index (DFI) vs pregnancy outcome. Fertil Steril 2008;90:1229-31.

29. Bungum M, Humaidan P, Axmon A, et al. Sperm DNA integrity assessment in prediction of assisted reproduction technology outcome. Hum Reprod 2007;22:174-9.

30. Oleszczuk K, Giwercman A, Bungum M. Sperm chromatin structure assay in prediction of in vitro fertilization outcome. Andrology 2016;4:290-6.

31. Erenpreiss J, Elzanaty S, Giwercman A. Sperm DNA damage in men from infertile couples. Asian J Androl 2008;10:786-90.

32. Simon L, Carrell DT. Sperm DNA damage measured by comet assay. Methods Mol Biol 2013;927:137-46.
Cite this article as: Evenson DP. Evaluation of sperm chromatin structure and DNA strand breaks is an important part of clinical male fertility assessment. Transl Androl Urol 2017;6(Suppl 4):S495-S500. doi: 10.21037/tau.2017.07.20 